\title{
A Dynamic Reaction Model of a Prey-predator System with Stage-structure for Predator
}

\author{
T. K. Kar (Corresponding author) \\ Department of Mathematics, Bengal Engineering and Science University \\ Shibpur Howrah-711103, West Bengal, India \\ E-mail: tkar1117@gmail.com \\ Saroj Kumar Chattopadhyay \\ Department of Mathematics, Sree Chaitanya College Habra, 24-pgs(N), West Bengal, India
}

\begin{abstract}
In this paper, we have considered a prey-predator model with stage-structure for predator and selective harvesting of prey species. A regulatory agency controls exploitation by imposing a tax per unit biomass of the prey species. The existence of steady states and their stability are studied. The problem of optimal harvesting policy is solved by using Pontryagin's maximal principle. It is also shown that time delay may cause a stable equilibrium to become unstable. Finally, some numerical simulations are carried out.
\end{abstract}

Keywords: Dynamic reaction, Stage-structure, Time delay, Optimal harvesting

\section{Introduction}

In a fully dynamic model of an open-access fishery, the level of fishing effort expands or contracts according as the net economic revenue to the fisherman is positive or negative. A model which includes this dynamic interaction between the net economic revenue and the fishing effort is called a dynamic reaction model. In the present paper, we study a dynamic reaction model of a prey-predator system where the selective harvesting of prey species is considered.

Taxation, license fees, lease of property rights, seasonal harvesting etc. are usually considered as possible governing instruments for the regulation of exploitation of biological resources which has become a problem of major concern now a days. Out of such regulatory options, taxation is considered to be superior because of its economic flexibility. Harvesting problems with taxation as a control instrument are studied by Chaudhuri \& Johnson (1990), Krishna et al. (1998), Chaudhuri (1998), Pradhan et al. (1999), Kar \& Chaudhuri (2003), Dubey et al. (2002) and references there in.

Prey-predator models play a crucial role in bioeconomics, that is the management of renewable resources. Chaudhuri (1986), Leung (1995), Dai and Tang (1998), Jerry \& Raissi (2001), Kar \& Chaudhuri (2004), Kar (2006) and some other authors have discussed the prey-predator system with harvesting. But they have not considered stage structure of species. Some of the stage structure models were considered by Aiello and Freedman (1990), Freedman and Gopalasammy (1986), Rozen (1987), Kar (2005), Kar and Pahari (2007) and some other authors. In general, stage-structured models exhibit much more complicated dynamics than ordinary models.

It is often that time delays are incorporated in the mathematical model of population biology. The dynamics of some stage-structured prey-predator model with discrete delay have been studied by Xu et al. (2004), Gourley et al. (2004), Zhang et al. (2000) and references there in. Xu et al. (2004) discussed the persistence and stability of a delayed prey-predator system. Gourley et al. (2004) studied a stage -structured prey-predator model and performed a systematic mathematical and computational study. Zhang et al. (2000) formulated a prey-predator model with stage-structured for prey and obtained the necessary and sufficient conditions for the system.

The rest of the paper is organized as follows: a stage-structured prey-predator model with discrete time delay and harvesting is established in the next section. Equilibria and their stability, optimal harvesting policy are discussed in the third section. Numerical simulation are provided in section 4. Finally the paper ends with a concluding remarks.

\section{Formulation of the model}

The ecological setup of the problem is as follows: for the prey-predator system only the prey is harvested and hence harvesting does not affect the growth of the predator population directly. Thus it is observed that the 
interaction between the harvesting agency and the predator is through the prey. Since the predator is unable to evolve a strategy for its survival, the regulating agency comes to the rescue of the predator through a suitable tax policy. We now formulate this problem mathematically. The population dynamics of the fishery resource is modelled by the equations

$$
\left.\begin{array}{l}
\frac{d N_{1}}{d t}=r_{1} N_{1}\left(1-\frac{N_{1}}{K}\right)-\alpha N_{1} N_{3}-q E N_{1}, \\
\frac{d N_{2}}{d t}=\beta N_{3}-r_{2} N_{2}, \\
\frac{d N_{3}}{d t}=-r_{3} N_{3}+m \alpha N_{1}(t-\tau) N_{3}(t-\tau)+\gamma N_{2}-d N_{3}^{2}, \\
\frac{d E}{d t}=\lambda\left[(p-\sigma) q N_{1}-c\right] E,
\end{array}\right\}
$$

with initial conditions

$$
\begin{aligned}
N_{i}(t) & =0 & & \text { for } t<0 \\
& =N_{i 0} & & \text { for } t=0 \\
E(t) & =E_{0} & & \text { for } t=0
\end{aligned}
$$

Here $N_{1}=N_{1}(t)$ is the size of the prey population at time $\mathrm{t}$,

$N_{2}=N_{2}(t)$ is the size of immature predator population at time $\mathrm{t}$,

$N_{3}=N_{3}(t)$ is the size of the mature predator population at time $\mathrm{t}$,

$E=E(t)$ is the harvesting effort at any time t.

All these populations are growing in a closed homogeneous environment. We consider the logistic growth of prey with birth rate $r_{1}$ and carrying capacity $\mathrm{k}$. At any time $\mathrm{t}(>0)$, the birth rate of immature predator is proportional to the density of existing mature predator with proportionality constant $\beta$. The death rates of mature and immature predators are $r_{3}, r_{2}$ respectively. The term $d N_{3}^{2}$ in the third equation is taken due to interspecific competition between the predators. The rate of conversion from immature predators to mature predators is $\quad \gamma ; \alpha$ is also a conversion factor, $\tau$ is discrete time delay which is the time interval between the moments when an individual prey is killed and when some fraction $m(0<m<1)$ of the corresponding biomass is added to the mature predator. The constant $q$ is catchability co-efficient, $p$ is the fixed price per unit of prey species, $c$ is the fixed cost of harvesting per unit of effort and $\lambda$ is called stiffness parameter used to measure the reaction of harvest effort.

To conserve the population in the prey-predator system the regulatory agency imposes a tax $\sigma>0$ per unit biomass of the prey. The harvesting agency and regulatory agency are actually to different components of the society at large. Hence the revenues earned by them are the revenues accrued to the society.

The net economic revenue to the society is

$$
p q E N_{1}-C E=\left[(p-\sigma) q E N_{1}-C E\right]+\sigma q E N_{1}
$$

which equals the net economic revenue to the harvesting agency plus the economic revenue to the regulatory agency.

\section{Equilibrium analysis}

From biological point of view we only concentrate on the interior equilibrium of the model where all species co-exist. The interior equilibrium of the model system (2.1) is $P\left(N_{1}^{*}, N_{2}^{*}, N_{3}^{*}, E^{*}\right)$ where 


$$
\begin{aligned}
& N_{1}^{*}=\frac{c}{(p-\sigma) q}, \\
& N_{2}^{*}=\frac{\beta}{d r_{2}}\left(\frac{\beta \gamma}{r_{2}}-r_{3}+m \alpha N_{1}^{*}\right), \\
& N_{3}^{*}=\frac{1}{d}\left(\frac{\beta \gamma}{r_{2}}-r_{3}+m \alpha N_{1}^{*}\right), \\
& E^{*}=\frac{1}{q}\left[r_{1}\left(1-\frac{N_{1}^{*}}{k}\right)-\alpha N_{3}^{*}\right] .
\end{aligned}
$$

From the expression of $N_{1}^{*}, N_{2}^{*}, N_{3}^{*}$ and $E^{*}$ it is easy to check that the interior equilibrium point exists provided the tax $\sigma$ lies in the range

$\sigma_{\min }<\sigma<\sigma_{\max }$

where

$$
\left.\begin{array}{l}
\sigma_{\text {min }}=p-\frac{m \alpha r_{2} c}{q\left(r_{2} r_{3}-\beta \gamma\right)}, \\
\sigma_{\max }=p-\frac{c r_{2}\left(d r_{1}+m k \alpha^{2}\right)}{k q\left(r_{1} r_{2} d-\alpha \beta \gamma+\alpha d r_{2} r_{3}\right)},
\end{array}\right\}
$$

and the parameters must satisfy the condition

$$
p>\frac{m \alpha r_{2} c}{q\left(r_{2} r_{3}-\beta \gamma\right)}>\frac{\left(d r_{1}+m k \alpha^{2}\right) c r_{2}}{k q\left(r_{1} r_{2} d-\alpha \beta \gamma+\alpha d r_{2} r_{3}\right)} \text {. }
$$

To discuss the stability at the interior equilibrium we construct the Jacobian of the model system (2.1) evaluated at the interior equilibrium $\mathrm{P}$, and write the following characteristic equation

$\left|\begin{array}{cccc}\frac{-r_{1} N_{1}^{*}}{k}-\mu & 0 & -\alpha N_{1}^{*} & -q_{1} N_{1}^{*} \\ 0 & -r_{2}-\mu & \beta & 0 \\ m \alpha N_{3}^{*} e^{-\mu \tau} & \gamma & -r_{3}+m \alpha N_{1}^{*} e^{-\mu \tau}-2 d N_{3}^{*}-\mu & 0 \\ \lambda E^{*}(p-\sigma) q & 0 & 0 & -\mu\end{array}\right|=0$

Or,

$$
X(\mu)+e^{-\mu \tau} Y(\mu)=0
$$

where

$$
\left.\begin{array}{l}
X(\mu)=\mu^{4}+x_{1} \mu^{3}+x_{2} \mu^{2}+x_{3} \mu+x_{4}, \\
Y(\mu)=y_{1} \mu^{3}+y_{2} \mu^{2}+y_{3} \mu+y_{4},
\end{array}\right\}
$$

$x_{1}=\frac{r_{1} N_{1}^{*}}{k}+r_{2}+r_{3}+2 d N_{3}^{*}$,

$x_{2}=\frac{r_{1} N_{1}^{*}}{k}\left(r_{2}+r_{3}+2 d N_{3}^{*}\right)+r_{2} r_{3}+2 d r_{2} N_{3}^{*}-\beta \gamma+\lambda(p-\sigma) q^{2} E^{*} N_{1}^{*}$,

$x_{3}=\frac{r_{1} N_{1}^{*}}{k}\left(r_{2} r_{3}+2 d r_{2} N_{3}^{*}-\beta \gamma\right)+\left(r_{2}+r_{3}+2 d N_{3}^{*}\right) \lambda(p-\sigma) q^{2} N_{1}^{*} E^{*}$,

$x_{4}=\left(r_{2} r_{3}+2 d r_{2} N_{3}^{*}-\beta \gamma\right) \lambda(p-\sigma) q^{2} E^{*} N_{1}^{*}$, 


$$
\begin{aligned}
& y_{1}=-m \alpha N_{1}^{*} \\
& y_{2}=-m \alpha\left(\frac{r_{1} N_{1}^{*}}{k}+r_{2}-\alpha N_{3}^{*}\right) N_{1}^{*}, \\
& y_{3}=-m \alpha\left[\lambda(p-\sigma) q^{2} E^{*} N_{1}^{* 2}+\frac{r_{1} r_{2} N_{1}^{* 2}}{k}-\alpha r_{2} N_{1}^{*} N_{3}^{*}\right], \\
& y_{4}=-m \alpha r_{2} \lambda(p-\sigma) q^{2} E^{*} N_{1}^{*^{2}} .
\end{aligned}
$$

We now discuss the stability of interior equilibrium.

\subsection{Stability analysis}

3.1.1 Case I: $\tau=0$.

In absence of discrete time delay we now investigate the stability of the model system around the interior equilibrium and optimal harvesting policy. In absence of delay i.e. when $\tau=0$, the system (2.1) is reduced to the following form:

$$
\begin{aligned}
& \frac{d N_{1}}{d t}=r_{1} N_{1}\left(1-\frac{N_{1}}{K}\right)-\alpha N_{1} N_{3}-q E N_{1}, \\
& \frac{d N_{2}}{d t}=\beta N_{3}-r_{2} N_{2}, \\
& \frac{d N_{3}}{d t}=-r_{3} N_{3}+m \alpha N_{1} N_{3}+\gamma N_{2}-d N_{3}^{2}, \\
& \frac{d E}{d t}=\lambda\left[(p-\sigma) q N_{1}-c\right] E,
\end{aligned}
$$

and the corresponding characteristic equation become

$$
\mu^{4}+\left(x_{1}+y_{1}\right) \mu^{3}+\left(x_{2}+y_{2}\right) \mu^{2}+\left(x_{3}+y_{3}\right) \mu+\left(x_{4}+y_{4}\right)=0 \text {. }
$$

By Routh - Hurwitz criterion it can be stated that the model is locally stable around the interior equilibrium point $\mathrm{P}$ if following set of conditions involving the parameters are satisfied.

$$
\left.\begin{array}{l}
\left(x_{1}+y_{1}\right)>0,\left(x_{1}+y_{1}\right)\left(x_{2}+y_{2}\right)-\left(x_{3}+y_{3}\right)>0 \\
\text { and }\left(x_{1}+y_{1}\right)\left(x_{2}+y_{2}\right)\left(x_{3}+y_{3}\right)-\left(x_{3}+y_{3}\right)^{2}-\left(x_{1}+y_{1}\right)^{2}\left(x_{4}+y_{4}\right)>0 .
\end{array}\right\}
$$

For finding the condition of global sability at $P\left(N_{1}^{*}, N_{2}^{*}, N_{3}^{*}, E^{*}\right)$ we construct the Lyapunov function

$$
v\left(N_{1}, N_{2}, N_{3}, E\right)=\sum_{i=1}^{3}\left[\left(N_{i}-N_{i}^{*}\right)-N_{i}^{*} \ln \frac{N_{i}}{N_{i}^{*}}\right]+\left(E-E^{*}\right)-E^{*} \ln \frac{E}{E^{*}} .
$$

The time derivative of $\mathrm{v}$ is given by

$$
\begin{aligned}
& \frac{d v}{d t}=\left(N_{1}-N_{1}^{*}\right)\left[r_{1}\left(1-\frac{N_{1}}{k}\right)-\alpha N_{3}-q E\right]+\left(N_{2}-N_{2}^{*}\right)\left(\frac{\beta N_{3}}{N_{2}}-r_{2}\right)+\left(N_{3}-N_{3}^{*}\right) \times \\
& {\left[-r_{3}+m \alpha N_{1}+\frac{\gamma N_{2}}{N_{3}}-d N_{3}\right]+\left(E-E^{*}\right) \lambda\left[(p-\sigma) q N_{1}-c\right] .}
\end{aligned}
$$

Now along the steady state solution of (3.9) 


$$
\begin{aligned}
& \frac{d v}{d t}=\left(N_{1}-N_{1}^{*}\right)\left[\frac{-r_{1}}{k}\left(N_{1}-N_{1}^{*}\right)-\alpha\left(N_{3}-N_{3}^{*}\right)\right]+\left(N_{2}-N_{2}^{*}\right) \frac{1}{N_{2}}\left[\beta\left(N_{3}-N_{3}^{*}\right)-r_{2}\left(N_{2}-N_{2}^{*}\right)\right] \\
& +\frac{\left(N_{3}-N_{3}^{*}\right.}{N_{3}}\left[-r_{3}\left(N_{3}-N_{3}^{*}\right)+m \alpha\left(N_{1} N_{3}-N_{1}^{*} N_{3}^{*}\right)+\gamma\left(N_{2}-N_{2}^{*}\right)-d\left(N_{3}^{2}-N_{3}^{* 2}\right)\right] \\
& +\left(E-E^{*}\right) \lambda\left[(p-\sigma) q\left(N_{1}-N_{1}^{*}\right)\right] .
\end{aligned}
$$

Again writing $N_{1} N_{3}-N_{1}^{*} N_{3}^{*}=N_{1}\left(N_{3}-N_{3}^{*}\right)+N_{3}^{*}\left(N_{1}-N_{1}^{*}\right)$ and rearranging the terms we get,

$$
\begin{aligned}
& \frac{d v}{d t}=-\frac{r_{1}}{k}\left(N_{1}-N_{1}^{*}\right)^{2}-\frac{r_{2}}{N_{2}}\left(N_{2}-N_{2}^{*}\right)^{2}-\left[\frac{r_{3}}{N_{3}}-m \alpha \frac{N_{1}}{N_{3}}+\frac{d}{N_{3}}\left(N_{3}+N_{3}^{*}\right)\right]\left(N_{3}-N_{3}^{*}\right)^{2} \\
& +\left(\frac{m \alpha N_{3}^{*}}{N_{3}}-\alpha\right)\left(N_{1}-N_{1}^{*}\right)\left(N_{3}-N_{3}^{*}\right)+\left(\frac{\beta}{N_{2}}+\frac{\gamma}{N_{3}}\right)\left(N_{2}-N_{2}^{*}\right)\left(N_{3}-N_{3}^{*}\right) \\
& +\lambda(p-\sigma) q\left(E-E^{*}\right)\left(N_{1}-N_{1}^{*}\right)=-X^{T} A X \\
& \text { where } X=\left[\begin{array}{ll}
N_{1}- & N_{1}^{*} \\
N_{2}- & N_{2}^{*} \\
N_{3}- & N_{3}^{*} \\
E- & E^{*}
\end{array}\right]
\end{aligned}
$$

and

$$
A=\left[\begin{array}{cccc}
\frac{r_{1}}{k} & 0 & \frac{1}{2}\left(\frac{m \alpha N_{3}^{*}}{N_{3}}-\alpha\right) & \frac{\lambda}{2}(p-\sigma) q \\
0 & \frac{r_{2}}{N_{2}} & \frac{1}{2}\left(\frac{\beta}{N_{2}}+\frac{\gamma}{N_{3}}\right) & 0 \\
\frac{1}{2}\left(\frac{m \alpha N_{3}^{*}}{N_{3}}-\alpha\right) & \frac{1}{2}\left(\frac{\beta}{N_{2}}+\frac{\gamma}{N_{3}}\right) & \frac{r_{3}}{N_{3}}-\frac{m \alpha N_{1}}{N_{3}}+\frac{d}{N_{3}}\left(N_{3}+N_{3}^{*}\right) & 0 \\
\frac{\lambda}{2}(p-\sigma) q & 0 & 0 & 0
\end{array}\right] \text {. }
$$

Now $d v / d t<0$ if the matrix $\mathrm{A}$ is positive definite i.e if the principal minors are positive. The principal minors of A, say, $M_{1}, M_{2}, M_{3}, M_{4}$ are given by $M_{1}=r_{1} / k, M_{2}=r_{1} r_{2} / k N_{2}$,

$$
M_{3}=\left|\begin{array}{ccc}
\frac{r_{1}}{k} & 0 & \frac{1}{2}\left(\frac{m \alpha N_{3}^{*}}{N_{3}}-\alpha\right) \\
0 & \frac{r_{2}}{N_{2}} & \frac{1}{2}\left(\frac{\beta}{N_{2}}+\frac{\gamma}{N_{3}}\right) \\
\frac{1}{2}\left(\frac{m \alpha N_{3}^{*}}{N_{3}}-\alpha\right) & \frac{1}{2}\left(\frac{\beta}{N_{2}}+\frac{\gamma}{N_{3}}\right) & \frac{r_{3}}{N_{3}}-\frac{m \alpha N_{1}}{N_{3}}+\frac{d}{N_{3}}\left(N_{3}+N_{3}^{*}\right)
\end{array}\right|,
$$

and 


$$
M_{4}=\frac{\lambda}{2}(p-\sigma) q\left|\begin{array}{cc}
\frac{r_{2}}{N_{2}} & \frac{1}{2}\left(\frac{\beta}{N_{2}}+\frac{\gamma}{N_{3}}\right) \\
\frac{1}{2}\left(\frac{\beta}{N_{2}}+\frac{\gamma}{N_{3}}\right) & \frac{r_{3}}{N_{3}}-\frac{m \alpha N_{1}}{N_{3}}+\frac{d}{N_{3}}\left(N_{3}+N_{3}^{*}\right)
\end{array}\right|
$$

These minors are all positive if

$$
r_{1}\left[4 r_{2} N_{2} N_{3}\left(r_{3}-m \alpha N_{1}+d N_{3}+d N_{3}^{*}\right)-\left(\beta N_{3}+\gamma N_{2}\right)^{2}\right]>k r_{2}^{2}\left(m \alpha N_{3}^{*}-\alpha N_{3}\right)^{2} \text {. }
$$

Thus the model (when $\tau=0$ ) is globally stable in the region given in(3.15).

\subsubsection{Optimal harvesting policy}

In this section we derive an optimal harvesting policy to maximize the total discounted net revenue from the harvesting biomass using $\sigma$ (the tax) as a control parameter and keeping the attention to the sustainable development of the prey-predator ecosystem. The net economic revenue $\pi\left(N_{1}, N_{2}, N_{3}, E, \sigma, t\right)=$ net revenue of harvesting agency + net economic revenue to the regulatory agency

$$
=(p-\sigma) q E N_{1}-c E+\sigma q E N_{1}=\left(p q N_{1}-c\right) E \text {. }
$$

Our objective is to maximize $\int_{0}^{\infty} e^{-\delta t}\left[p q N_{1}(t)-c\right] E(t) d t$, where $\delta$ is instantaneous annual rate of discount and the optimization problem is subjected to the model system (3.9). The control variable $\sigma(t)$ is subjected to constraints $\sigma_{\min } \leq \sigma(t) \leq \sigma_{\max }$.

Using Pontryagin's maximal principle (1964), the associated Hamiltonian

$H\left(N_{1}(t), N_{2}(t), N_{3}(t), E(t), \sigma(t), t\right)$ is given by

$$
\begin{gathered}
H=e^{-\delta t}\left[p q N_{1}(t)-c\right] E(t)+\lambda_{1}\left[r_{1} N_{1}(t)\left(1-\frac{N_{1}(t)}{k}\right)-\alpha N_{1}(t) N_{3}(t)-q E(t) N(t)\right] \\
+\lambda_{2}\left[\beta N_{3}(t)-r_{2} N_{2}(t)\right]++\lambda_{3}\left[-r_{3} N_{3}(t)+m \alpha N_{1}(t) N_{3}(t)+\gamma N_{2}(t)-d N_{3}^{2}(t)\right] \\
+\lambda_{4}\left[\lambda\left\{(p-\sigma) q N_{1}(t)-c\right\}\right] E(t),
\end{gathered}
$$

where $\lambda_{i}(t)(i=1,2,3,4)$ are adjoint variables.

The condition for a singular control to be optimal is $\partial H / \partial \sigma=0$ which in turn implies

$$
\lambda_{4}(t)=0
$$

The adjoint equations are $\frac{d \lambda_{1}}{d t}=-\frac{\partial H}{\partial N_{1}}, \frac{d \lambda_{2}}{d t}=-\frac{\partial H}{\partial N_{2}}, \frac{d \lambda_{3}}{d t}=-\frac{\partial H}{\partial N_{3}}, \frac{d \lambda_{4}}{d t}=-\frac{\partial H}{\partial E}$.

These equations when evaluated at the interior equilibrium, give

$$
\begin{aligned}
& \frac{d \lambda_{1}}{d t}=-p q E e^{-\delta t}+\lambda_{1} \frac{r_{1} N_{1}}{K}-m \alpha \lambda_{3} N_{3}, \\
& \frac{d \lambda_{2}}{d t}=\lambda_{2} r_{2}-\lambda_{3} \gamma, \\
& \frac{d \lambda_{3}}{d t}=\lambda_{1} \alpha N_{1}-\lambda_{2} \beta-\lambda_{3}\left(-r_{3}+m \alpha N_{1}-2 d N_{3}\right),
\end{aligned}
$$




$$
\frac{d \lambda_{4}}{d t}=-e^{-\delta t}\left(p q N_{1}-c\right)+\lambda_{1} q N_{1}
$$

From (3.22) we get

$$
\lambda_{1}=e^{-\delta t}\left(p-c / q N_{1}\right)
$$

In order to obtain an optimal equilibrium solution we get from (3.23) and (3.19) that

$$
-\delta e^{-\delta t}\left(p-c / q N_{1}^{*}\right)=-p q E^{*} e^{-\delta t}+\frac{r_{1} N_{1}^{*}}{k} e^{-\delta t}\left(p-c / q N_{1}^{*}\right)+m \alpha N_{3}^{*} \lambda_{3} .
$$

or, $\quad \lambda_{3}=A_{3} e^{-\delta t}$,

where

$$
A_{3}=\frac{1}{m \alpha N_{3}^{*}}\left[-p q E^{*}+\frac{r_{1} N_{1}^{*}}{K}\left(p-c / q N_{1}^{*}\right)+\delta\left(p-c / q N_{1}^{*}\right)\right] \text {. }
$$

From (3.20) and (3.24) we get, $\frac{d \lambda_{2}}{d t}=\lambda_{2} r_{2}-A_{3} \gamma e^{-\delta t}$ or, $\frac{d \lambda_{2}}{d t}-\lambda_{2} r_{2}=-A_{3} \gamma e^{-\delta t}$ which is a linear differential equation with integrating factor $e^{-r_{2} t}$. Thus we write it as $\frac{d}{d t}\left(\lambda_{2} e^{-r_{2} t}\right)=-A_{3} \gamma e^{-\left(\delta+r_{2}\right) t}$. To have a bounded solution we integrate it and make the arbitrary constant of integration zero, so that

$$
\lambda_{2}=A_{3} \frac{\gamma}{\delta+r_{2}} e^{-\delta t}
$$

Using (3.23) \& (3.24) in (3.21) we obtain

$-A_{3} \delta e^{-\delta t}=\alpha N_{1}^{*} e^{-\delta t}\left(p-c / q N_{1}^{*}\right)-\frac{A_{3} \beta \gamma}{\delta+r_{2}} e^{-\delta t}-A_{3} e^{-\delta t}\left(-r_{3}+m \alpha N_{1}^{*}-2 d N_{3}^{*}\right)$.

From which we have

$$
p-\frac{c}{q N_{1}^{*}}=\frac{A_{3}}{\alpha N_{1}^{*}}\left[-\delta+\frac{\beta \gamma}{\delta+r_{2}}+m \alpha N_{1}^{*}-2 d N_{3}^{*}-r_{3}\right] .
$$

This provides an equation to the singular path and gives the optimal equilibrium levels of the populations $N_{1}^{*}=N_{1 \delta}, N_{2}^{*}=N_{2 \delta}, N_{3}^{*}=N_{3 \delta}$. Then optimal equilibrium of harvesting effort and tax can be obtained as $E_{\delta}=\frac{1}{q}\left[r_{1}\left(1-N_{1 \delta} / k\right)-\alpha N_{3 \delta}\right]$ and $\sigma_{\delta}=p-c / q N_{1 \delta}$.

Now $\lambda_{i}(t) e^{\delta t}$ (i = 1,2,3,4) represent shadow prices along the singular path. From (3.23), (3.24) \& (3.26) it may be concluded that these shadow prices may remain constant over time interval in an optimal equilibrium when they satisfy strictly transversality condition at $\infty$. Further they remain bounded when $t \rightarrow \infty$. From (3.23), $\lambda_{1}(t) q N_{1}^{*}=e^{-\delta t}\left(p q N_{1}^{*}-c\right)=e^{-\delta t} \frac{\partial \pi}{\partial E} \quad$ which implies that the total users cost of harvest per unit effort is equal to the discounted values of the future price at the steady state level.

3.2 Case II: $\tau>0$. We now discuss the stability of the model system (2.1) in the presence of delay. Let $\mu=i \omega$ be a root of (3.5), where $\omega$ is a real number. Putting $\mu=i \omega$ in (3.5) and separating real and imaginary parts we get,

$$
\begin{aligned}
& \omega_{4}-x_{2} \omega^{2}+x_{4}=\left(\omega^{3} y_{1}-\omega y_{3}\right) \sin \omega \tau+\left(\omega^{2} y_{2}-y_{4}\right) \cos \omega \tau, \\
& \omega_{3} x_{1}-\omega x_{3}=\left(\omega^{2} y_{2}-y_{4}\right) \sin \omega \tau-\left(\omega^{3} y_{1}-\omega y_{3}\right) \cos \omega \tau .
\end{aligned}
$$


Squaring and adding these two equations we get,

$$
\omega^{8}+B_{1} \omega^{6}+B_{2} \omega^{4}+B_{3} \omega^{2}+B_{4}=0
$$

$$
\left.\begin{array}{l}
\text { where } \quad B_{1}=x_{1}^{2}-2 x_{2}-y_{1}^{2} ; B_{2}=x_{2}^{2}+2 x_{4}-2 x_{1} x_{3}+2 y_{1} y_{3}-y_{2}^{2}, \\
B_{3}=x_{3}^{2}-2 x_{2} x_{4}+2 y_{2} y_{4}-y_{3}^{2} ; B_{4}=x_{4}^{2}-y_{4}^{2} .
\end{array}\right\}
$$

If $B_{3}<0$ and $B_{4}<0$ i.e. $x_{3}^{2}-2 x_{2} x_{4}+2 y_{2} y_{4}-y_{3}^{2}<0$ and $x_{4}^{2}-y_{4}^{2}<0$, then (3.30) has a unique positive root, say, $\omega_{0}$ and then (3.5) has a pair of imaginary roots $\pm i \omega_{0}$. Eliminating $\sin \omega \tau$ from (3.28) and (3.29) it can be obtained that

$$
\cos \omega \tau=\frac{\left(\omega^{4}-x_{2} \omega^{2}+x_{4}\right)\left(y_{2} \omega^{2}-y_{4}\right)\left(\omega^{3} y_{1}-\omega y_{3}\right)\left(\omega^{3} x_{1}-\omega x_{3}\right)}{\left(\omega^{3} y_{1}-\omega y_{3}\right)^{2}+\left(\omega^{2} y_{2}-y_{4}\right)^{2}} .
$$

Then $\tau_{k}$ corresponding to $\omega=\omega_{0}$ is given by

$$
\tau_{k}=\frac{1}{\omega_{0}} \cos ^{-1}\left[\frac{\left(\omega_{0}^{4}-x_{2} \omega_{0}^{2}+x_{4}\right)\left(\omega_{0}^{2} y_{2}-y_{4}\right)-\left(y_{1} \omega_{0}^{3}-y_{3} \omega_{0}\right)\left(x_{1} \omega_{0}^{3}-x_{3} \omega_{0}\right)}{\left(\omega_{0}^{3} y_{1}-y_{3} \omega_{0}\right)^{2}+\left(y_{2} \omega_{0}^{2}-y_{4}\right)^{2}}\right]+\frac{2 k \pi}{\omega_{0}}
$$

where $k=0,1,2, \ldots \ldots$

Thus by Butler's Lemma we conclude that the model system (2.1) is stable around the interior equilibrium for $\tau<\tau_{0}$ as $k=0$.

Now differentiating the characteristic equation (3.5) we can obtain,

$$
\begin{aligned}
\left(\frac{d \mu}{d \tau}\right)^{-1} & =\frac{4 \mu^{3}+3 x_{1} \mu^{2}+2 x_{2} \mu+x_{3}}{-\mu\left(\mu^{4}+x_{1} \mu^{3}+x_{2} \mu^{2}+x_{3} \mu+x_{4}\right)}+\frac{3 y_{1} \mu^{2}+2 y_{2} \mu+y_{3}}{\mu\left(y_{1} \mu^{3}+y_{2} \mu^{2}+y_{3} \mu+y_{4}\right)}-\frac{\tau}{\mu} \\
& =\frac{4 \mu^{4}+3 x_{1} \mu^{3}+2 x_{2} \mu^{2}+x_{3} \mu}{-\mu^{2}\left(\mu^{4}+x_{1} \mu^{3}+x_{2} \mu^{2}+x_{3} \mu+x_{4}\right)}+\frac{3 y_{1} \mu^{3}+2 y_{2} \mu^{2}+y_{3} \mu}{\mu^{2}\left(y_{1} \mu^{3}+y_{2} \mu^{2}+y_{3} \mu+y_{4}\right)}-\frac{\tau}{\mu} \\
& =\frac{3 \mu^{4}+2 x_{1} \mu^{3}+x_{2} \mu^{2}-x_{4}}{-\mu^{2}\left(\mu^{4}+x_{1} \mu^{3}+x_{2} \mu^{2}+x_{3} \mu+x_{4}\right)}+\frac{2 y_{1} \mu^{3}+y_{2}^{2}-y_{4}}{\mu^{2}\left(y_{1} \mu^{3}+y_{2} \mu^{2}+y_{3} \mu+y_{4}\right)}-\frac{\tau}{\mu} .
\end{aligned}
$$

Thus

$$
\begin{aligned}
& \left.\left(\frac{d \mu}{d \tau}\right)^{-1}\right|_{\mu=i \omega_{0}}= \\
& \frac{\left(3 \omega_{0}^{4}-x_{2} \omega_{0}^{2}-x_{4}\right)-2 i x_{1} \omega_{0}^{3}}{\omega_{0}^{2}\left[\left(\omega_{0}^{4}-x_{2} \omega_{0}^{2}+x_{4}\right)-i\left(x_{1} \omega_{0}^{3}-x_{3} \omega_{0}\right)\right]}+\frac{\left(y_{2} \omega_{0}^{2}+y_{4}\right)+i\left(2 y_{1} \omega_{0}^{3}\right)}{\omega_{0}^{2}\left[-\left(y_{2} \omega_{0}^{2}-y_{4}\right)-i\left(y_{1} \omega_{0}^{3}-y_{3} \omega_{0}\right)\right]}-\frac{\tau}{i \omega_{0}} . \\
& \left.\therefore \operatorname{Re}\left(\frac{d \mu}{d \tau}\right)^{-1}\right|_{\mu=i \omega_{0}} \quad \frac{\left(y_{4}-y_{2} \omega_{0}^{2}\right)\left(y_{4}-y_{2} \omega_{0}^{2}\right)+2 y_{1} \omega_{0}^{3}\left(y_{3} \omega_{0}-y_{1} \omega_{0}^{3}\right)}{\left.\left(y_{4}-y_{2} \omega_{0}^{2}\right)^{2}+\left(y_{1} \omega_{0}^{3}-y_{3} \omega_{0}\right)^{2}-x_{2} \omega_{0}^{2}-x_{4}\right)\left(\omega_{0}^{4}-x_{2} \omega_{0}^{2}+x_{4}\right)+2 x_{1} \omega_{0}^{3}\left(x_{1} \omega_{0}^{3}-x_{3} \omega_{0}\right)}\left(\omega_{0}^{4}-x_{2} \omega_{0}^{2}+x_{4}\right)^{2}+\left(x_{1} \omega_{0}^{3}-x_{3} \omega_{0}\right)^{2}
\end{aligned}
$$

(using (3.30)) 
where $\xi^{2}=\left(\omega_{0}^{4}-x_{2} \omega_{0}^{2}+x_{4}\right)^{2}+\left(x_{1} \omega_{0}^{3}-x_{3} \omega_{0}\right)^{2}=\left(y_{4}-y_{2} \omega_{0}^{2}\right)^{2}+\left(y_{1} \omega_{0}^{3}-y_{3} \omega_{0}\right)^{2}$.

Now on the basis of existence of positive root of (3.30), sign of $\operatorname{Re}\left(\frac{d \mu}{d \tau}\right)^{-1}$ is positive if

$x_{1}^{2}-2 x_{2}-y_{1}^{2}>0$

that

is

if

$\left(\frac{r_{1}^{2}}{k^{2}}-m^{2} \alpha^{2}\right) N_{1}^{2}+4 d^{2} N_{3}^{2}+4 r_{3} d N_{3}-2 \lambda(p-\sigma) q^{2} E N_{1}+r_{2}^{2}+r_{3}^{2}+2 \beta \gamma>0$

when evaluated at interior equilibrium.

In that case, sign $\left\{\left(\frac{d}{d \tau}(\operatorname{Re} \mu)\right\}=\operatorname{sign}\left\{\operatorname{Re}\left(\frac{d \mu}{d \tau}\right)^{-1}\right\}\right.$.

That is transversality condition holds and Hopf bifurcation occurs at $\tau=\tau_{0}$.

\section{Numerical simulation}

We consider the following set of values of the parameters for our numerical simulations:

$r_{1}=2.5, k=100, \alpha=0.5, q=0.5, b=0.5, r_{2}=0.1, r_{3}=3, m=0.1, \gamma=0.5, d=0.05, p=15$, $c=7.5, \delta=0.01$ in appropriate units.

Then for the above values of parameters optimal tax becomes $\sigma=13.73742$, and corresponding stable optimal equilibrium is $(11.87044,9.405178,1.880436,2.525543)$.

$<$ Figure $1>$

$<$ Figure $2>$

$<$ Figure $3>$

$<$ Figure $4>$

$<$ Figure $5>$

$<$ Figure $6>$

\section{Concluding remarks}

Attempts are made to understand the effect of delay on the stability of the considered system. Stability analysis shows that the discrete time delay may cause for the stability switch of the model system, and phenomenon of Hopf bifurcation occurs as the time delay crosses through a certain threshold.

In the present work we have elaborated a bioeconomic model which is realistic because we force the fishing effort to remain under control by imposing a tax to keep ecological balance. Another important feature of the present model is that it assumes a fully dynamic interaction between fishing effort and the perceived rent in the case of a prey-predator fishery.

\section{References}

Aiello, W. G. \& Freedman, H. I. (1990). A time delay model of single species growth with stage structure, Math. Biosci. 101, 139.

Chaudhuri, K. S. (1986). A bioeconomic model of harvesting: a multispecies fishery. Ecol. Model. 32, 267-279.

Chaudhuri, K. S. (1998). Dynamic optimization of combined harvesting of two species fishery, Ecological Modelling, 41, 17-25.

Chaudhuri, K. S. and Johnson, T. (1990). Bioeconomic dynamics of a fishery Modeled as and S-system, Mathematical Biosciences, 49, 231-249.

Dai, G. \& Tang, M.(1998). Coexistence region and global dynamics of harvested predator-prey system, SIAM J. Appl. Math. 13, 193-210.

Dubey, B. Chandra, P. \& Sinha, P. (2002). A resource dependent fishery model with optimal harvesting policy, Journal of biological System, 10(1), 1-13. 
Freedman, H. I. \& Gopalsammy, K. (1986). Global stability in time-delayed single species dynamics, Bull. Math. Biol. 48, 485.

Gourley, S. A. \& Kuang, Y. A. (2004). Stage structured prey-predator model and its dependence on maturation delay and death rate. Journal of mathematical Biology, 49, 188-200.

Jerry, M. \& Raissi, N. (2001). A policy of fisheries Management based on continuous fishing effort, J. Biol. Syst. 9, 247-254.

Kar, T. K. \& Chaudhuri, K. S.(2003). Regulation of a prey -predator fishery by taxation: a dynamic reaction model, Journal of Biological Systems, 11(2), 173-187.

Kar, T. K. \& Chaudhuri, K.S. (2004). Harvesting in a two prey one predator fishery: A bioeconomic model, ANZIAM J. 45, 443-456.

Kar, T. K. \& Phari, U.K. Modelling and analysis of a prey-predator system with stage-structure and harvesting, Nonlinear Analysis: Real World Applications, 8, 601-609.

Kar, T. K. (2006). A model for fishery resource with reserve area and facing prey predator interactions, Canadian Applied Mathematics Quarterly, 14(4), 385-399.

Kar, T.K. Stability and optimal harvesting of prey-predator model with stage structure for predator. Applications Mathematica, 32(3), 279-291.

Krishna, S. V., Srinivasu, P. D. N. \& Kaymakcalan, B. (1998). Conservation of ecosystem through optimal taxation, Bulletin of Mathematical Biology, 60, 569-584.

Leung, A.(1995). Optimal harvestting coefficient control of steady state prey-predator diffusive Volterra-Lotka systems, Appl. Math. Optim, 31, 219.

Pontryagin, L.S. Boltyansk, V.G. Gamkrelidre, K.V.\& Mischenko, E. F. The Mathematical Theory of Optimal Processes, Pergamon Press, London, 1964.

Pradhan, T. \& Chaudhuri, K. S. (1999). A dynamic reaction model of a two-species fishery with taxation as a control instrument: a Capital theoretic analysis, Ecological Modelling, 121, 1-16.

Rozen, G. (1987). Time delays produced by essential non-linearity in population growth models Bull. Math. Biol. 49, 253.

Xu, R., Chaplin, M. A. \& Davidson, F.A. (2004). Persistence and stability of a stage-structured predator-prey model with time delays, Applied Mathematics and Computation, 150, 259-277.

Zhang, X. Chen, L. \& Neumann, U. A. (2000). The stage- structured predator-prey model and optimal harvesting policy, Mathematical Biosciences, 168, 201-210.

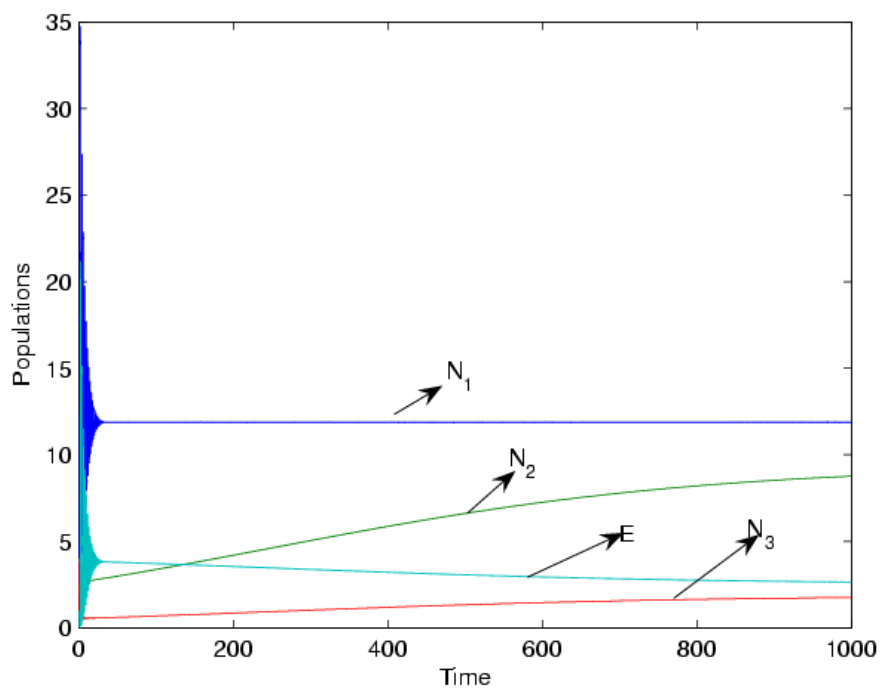

Figure 1. Solution curves corresponding to the optimal tax $\sigma=13.73742$, beginning with

$$
N_{1}=2.5, N_{2}=2, N_{3}=4, E=1 \text {. }
$$




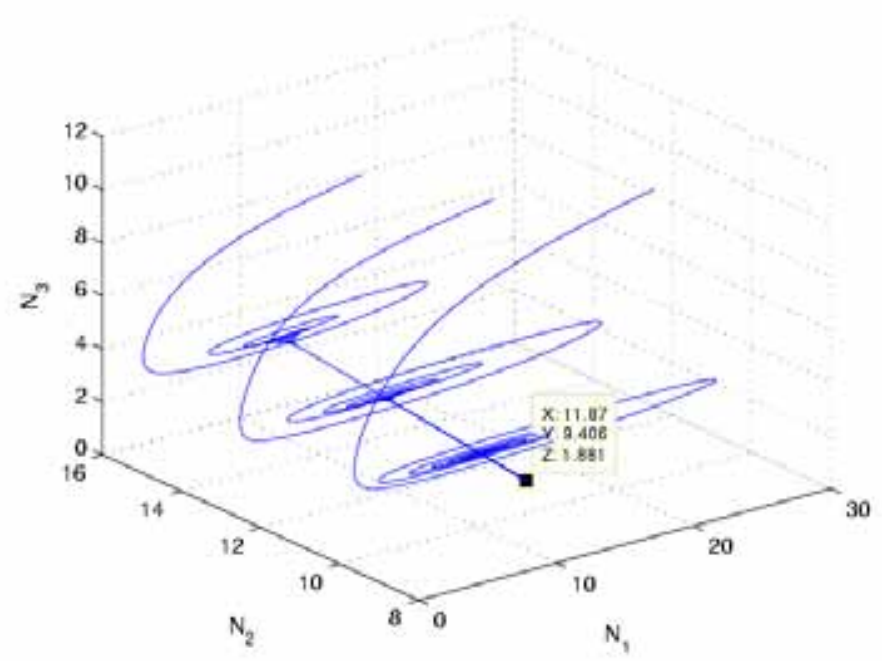

Figure 2. Phase space trajectories of $N_{1} N_{2} \& N_{3}$, corresponding to the optimal tax $\sigma=13.73742$ begin with different initial levels. Trajectories clearly indicate that the optimal equilibrium $(11.87,9.406,1.881)$ is asymptotically stable.

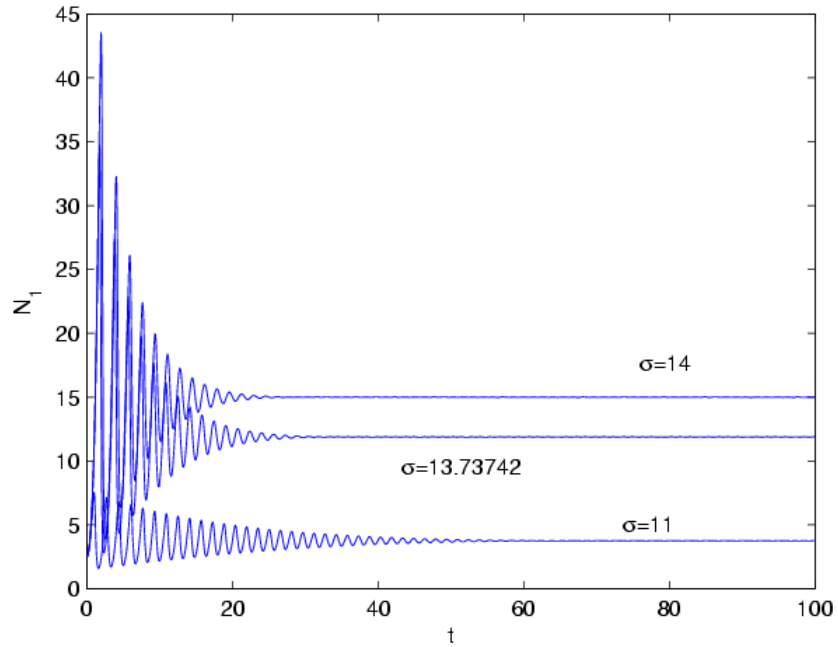

Figure 3. Variation of prey population with time for different tax levels 


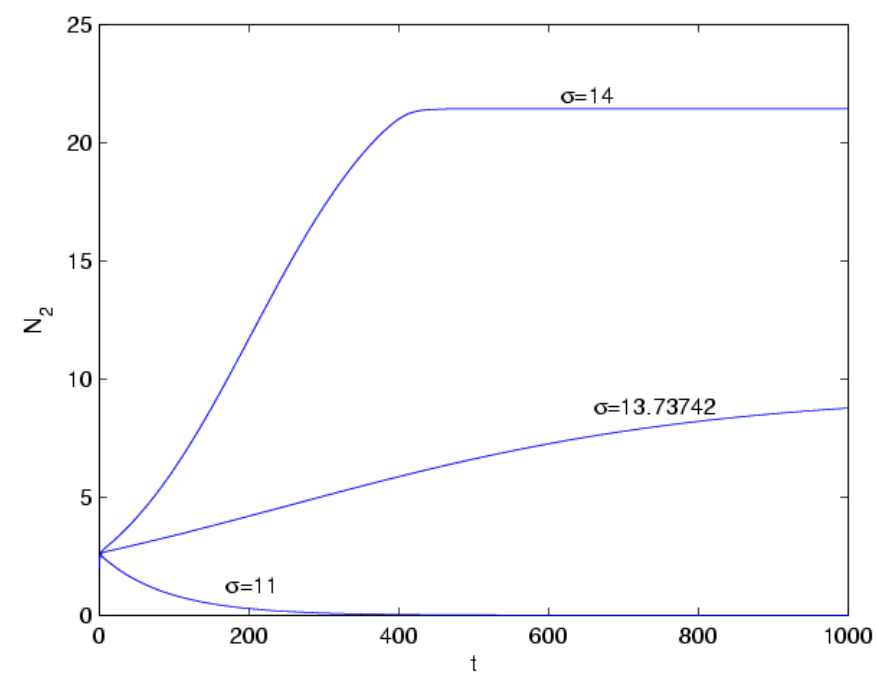

Figure 4. Variation of immature predator population with time different tax levels

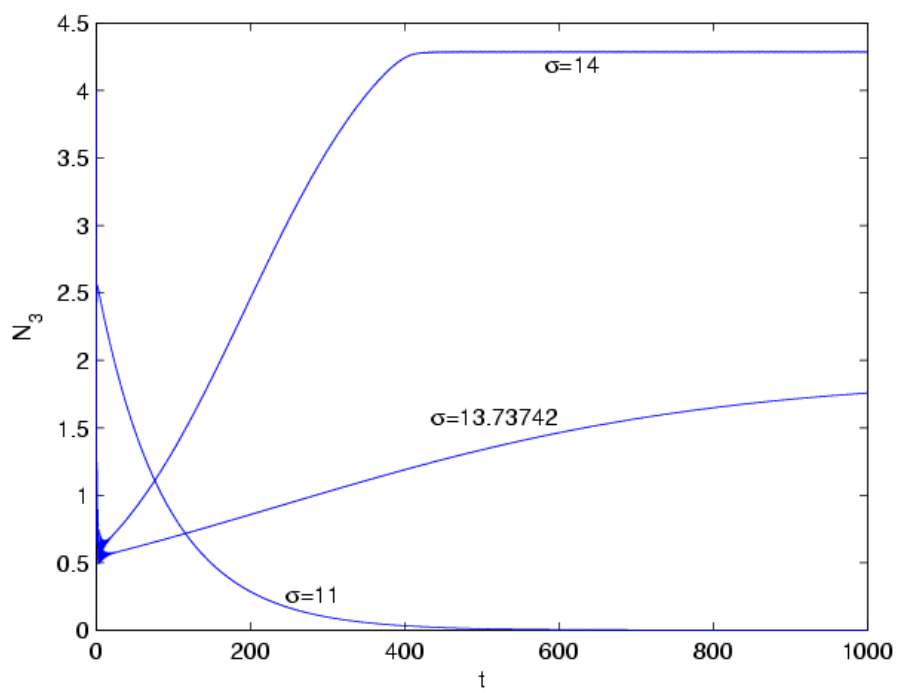

Figure 5. Variation of mature predator population with different tax levels 


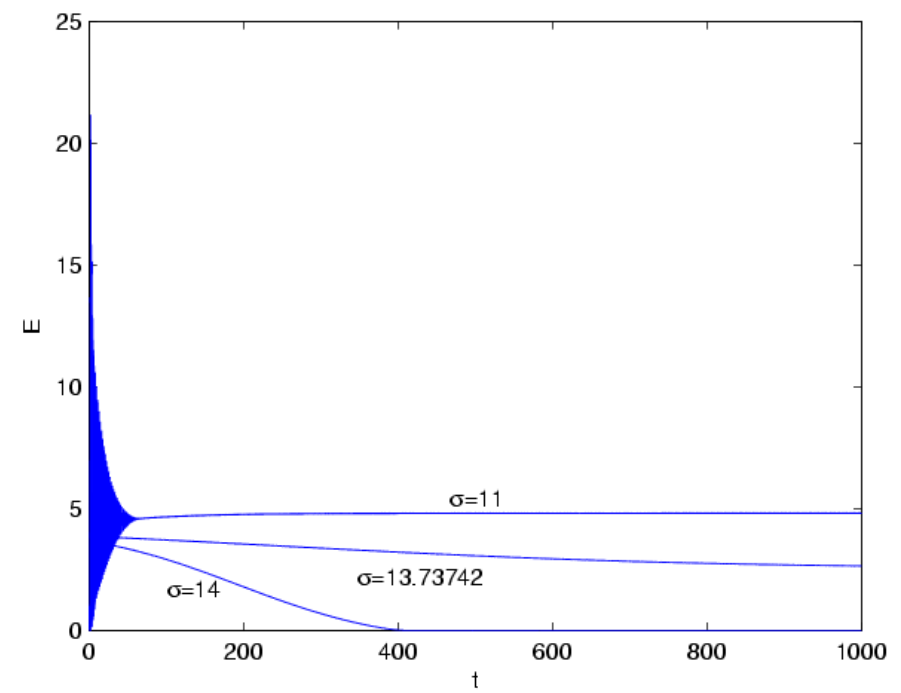

Figure 6. Variation of harvesting effort with different tax levels 